\title{
Cardiovascular Magnetic Resonance in Peripartum Cardiomyopathy: Comparison with Idiopathic Dilated Cardiomyopathy
}

\author{
Joanna Petryka-Mazurkiewicz 1,2,*®D, Karolina Kryczka ${ }^{1}$, tukasz Mazurkiewicz ${ }^{3}$, Barbara Miłosz-Wieczorek ${ }^{2}$, \\ Mateusz Śpiewak $^{2}$, Magdalena Marczak ${ }^{2}$, Jan Henzel ${ }^{1}{ }^{1}$, Jacek Grzybowski ${ }^{3}$, Marcin Demkow ${ }^{1}$ \\ and Zofia Dzielińska ${ }^{1}$
}

Citation: Petryka-Mazurkiewicz, J.; Kryczka, K.; Mazurkiewicz, Ł.; Miłosz-Wieczorek, B.; Śpiewak, M.; Marczak, M.; Henzel, J.; Grzybowski, J.; Demkow, M.; Dzielińska, Z. Cardiovascular Magnetic Resonance in Peripartum Cardiomyopathy: Comparison with Idiopathic Dilated Cardiomyopathy. Diagnostics 2021, 11, 1752. https://doi.org/10.3390/ diagnostics11101752

Academic Editor: Andrea D. Annoni

Received: 27 August 2021

Accepted: 21 September 2021

Published: 24 September 2021

Publisher's Note: MDPI stays neutral with regard to jurisdictional claims in published maps and institutional affiliations.

Copyright: (c) 2021 by the authors. Licensee MDPI, Basel, Switzerland. This article is an open access article distributed under the terms and conditions of the Creative Commons Attribution (CC BY) license (https:/ / creativecommons.org/licenses/by/ $4.0 /)$.
1 Department of Coronary and Structural Heart Diseases, National Institute of Cardiology, 04-628 Warsaw, Poland; kkryczka@ikard.pl (K.K.); jhenzel@ikard.pl (J.H.); mdemkow@ikard.pl (M.D.); zdzielinska@ikard.pl (Z.D.)

2 Magnetic Resonance Unit, National Institute of Cardiology, 04-628 Warsaw, Poland; bmilosz@ikard.pl (B.M.-W.); mspiewak@ikard.pl (M.Ś.); mmarczak@ikard.pl (M.M.)

3 Department of Cardiomyopathy, National Institute of Cardiology, 04-628 Warsaw, Poland; lmazurkiewicz@ikard.pl (Ł.M.); jgrzybowski@ikard.pl (J.G.)

* Correspondence: j.petryka@ikard.pl; Tel.: +48-504273409

Abstract: Background: Peripartum (PPCM) and dilated (DCM) cardiomyopathies are distinct forms of cardiac disease that share certain aspects in clinical presentation. Aim: We hypothesized that different cardiac structural changes underlie PPCM and DCM, and we aimed to investigate them with cardiovascular magnetic resonance (CMR). Methods: We included 21 PPCM patients (30.5 \pm 5.9 years) and 30 female DCM patients (41.5 \pm 16.8 years) matched for left ventricular ejection fraction. Biventricular and biatrial volumetric and functional parameters were assessed along with ventricular and atrial strain indices based on feature-tracking techniques. The presence of late gadolinium enhancement (LGE) was also assessed. Results: In PPCM, the left ventricular (LV) stroke volume index was lower $(p=0.04)$, right atrial (RA) minimal and pre-systolic volumes were higher $(p<0.01$ and $p=0.02$, respectively), and the total RA ejection fraction was lower $(p=0.02)$ in comparison to DCM. Moreover, in PPCM, the LV global longitudinal strain $(p=0.03)$, global circumferential strain rate $(p=0.04)$, and global longitudinal strain rate $(p<0.01)$ were less impaired than in DCM. Both PPCM and DCM patients with LGE had more dilated ventricles and more impaired LV and left atrial function than in PPCM and DCM patients without LGE. Conclusions: Subtle differences appear on CMR between PPCM and DCM. Most importantly, the RA is larger and more impaired, and LV global longitudinal strain is less reduced in PPCM than in DCM. Furthermore, similarly to DCM, PPCM patients with LGE have more dilated and impaired ventricles than patients without LGE.

Keywords: peripartum cardiomyopathy; dilated cardiomyopathy; cardiovascular magnetic resonance; feature tracking; late gadolinium enhancement

\section{Introduction}

Peripartum cardiomyopathy (PPCM) and dilated cardiomyopathy (DCM) are two cardiac conditions that share some aspects in clinical presentation, such as dilated ventricles, reduced systolic function, and arrhythmias [1]. However, PPCM is a rare cardiac condition that presents itself during late pregnancy or during the early postpartum period, with a clinical course that is distinct from that of idiopathic DCM in young women [2]. Patients with PPCM typically present in their early 30s, while patients with DCM usually present later in life, unless genetically predisposed. While heart muscle function in DCM never fully recovers but slowly deteriorates further, the clinical course of PPCM may vary between rapid cardiac deterioration or complete recovery. Between $20 \%$ and $60 \%$ of patients with PPCM recover LV function [3,4]. However, the mortality rate of PPCM remains high-from 
$11 \%$ to $32 \%[3,4]$. Patients with DCM have a decreasing survival rate, with $90 \%$ of patients surviving at 1 year, $50 \%$ at 5 years, and 33\% at 10 years [5]. Already in the 1960 s of the twentieth century, it was observed that some cases of PPCM cluster in families [6]. A genetic cause for DCM and PPCM has been demonstrated before [7]. It has been reported that approximately $15 \%$ to $20 \%$ of PPCM patients carry cardiomyopathy-causing mutations, mainly in the TTN, MYH7, and SCN5A genes [8]. Mutations in these genes are associated with DCM, as well [8].

Cardiac magnetic resonance (CMR) imaging is a recognized radiation-free technique used to assess cardiac structure and function [9]. Cine CMR is a highly accurate and reproducible technique for the determination of cardiac volumes, function, and mass [10]. Furthermore, CMR feature tracking (CMR-FT) provides detailed analysis of biventricular and biatrial mechanics. Late gadolinium enhancement (LGE) CMR is considered the gold standard for assessing focal myocardial fibrosis. Although CMR imaging is used for the diagnosis and detection of myocardial damage in various heart diseases, there are limited data on its diagnostic or prognostic usefulness in PPCM [11].

On the basis of differences in disease onset and progression, we hypothesized that different cardiac structural changes underlie PPCM and DCM, and we aimed to investigate them with CMR.

\section{Materials and Methods}

This study was approved by the Institutional Ethics Committee, and written informed consent to participate in the study was obtained from all subjects.

\subsection{Study Population}

The study cohort consisted of subjects with PPCM and female subjects with DCM matched for left ventricular ejection fraction, who underwent clinically indicated CMR in our center between 2014 and 2020. PPCM was defined according to the ESC criteria as cardiomyopathy presenting towards the end of pregnancy or in the months following delivery where no other cause of heart failure was found [12]. DCM was diagnosed based on the revised ESC definition that includes the presence of left ventricular (LV) or biventricular dilatation and systolic dysfunction in the absence of abnormal loading conditions (hypertension and valve disease) or the presence of coronary artery disease sufficient to cause global systolic impairment [1].

\subsection{CMR Examination}

Cardiovascular magnetic resonance imaging was performed by using a 1.5-T scanner (Sonata and Avanto fit, Siemens, Erlangen, Germany). Cine images were acquired by breath-hold, electrocardiographic-gated, segmented k-space steady-state free-precession (SSFP) technique using 25 phases per cardiac cycle. LGE images were obtained in the longaxis and short-axis imaging planes by using a breath-hold segmented inversion recovery sequence implemented 10-15 min after intravenous administration of $0.1 \mathrm{mmol} / \mathrm{kg}$ of gadobutrol (Gadovist, Bayer, Berlin, Germany). The presence of left ventricular LGE was initially visually assessed by two independent experienced observers (JPM and LM). If positive, quantification was performed using the using CVi42 software (Circle Cardiovascular Imaging Inc., Calgary, AB, Canada) with a signal intensity threshold of $>6$ standard deviations above the remote myocardium (Figure 1) [13]. The extent of LGE was presented as a percentage of the total LV mass. 


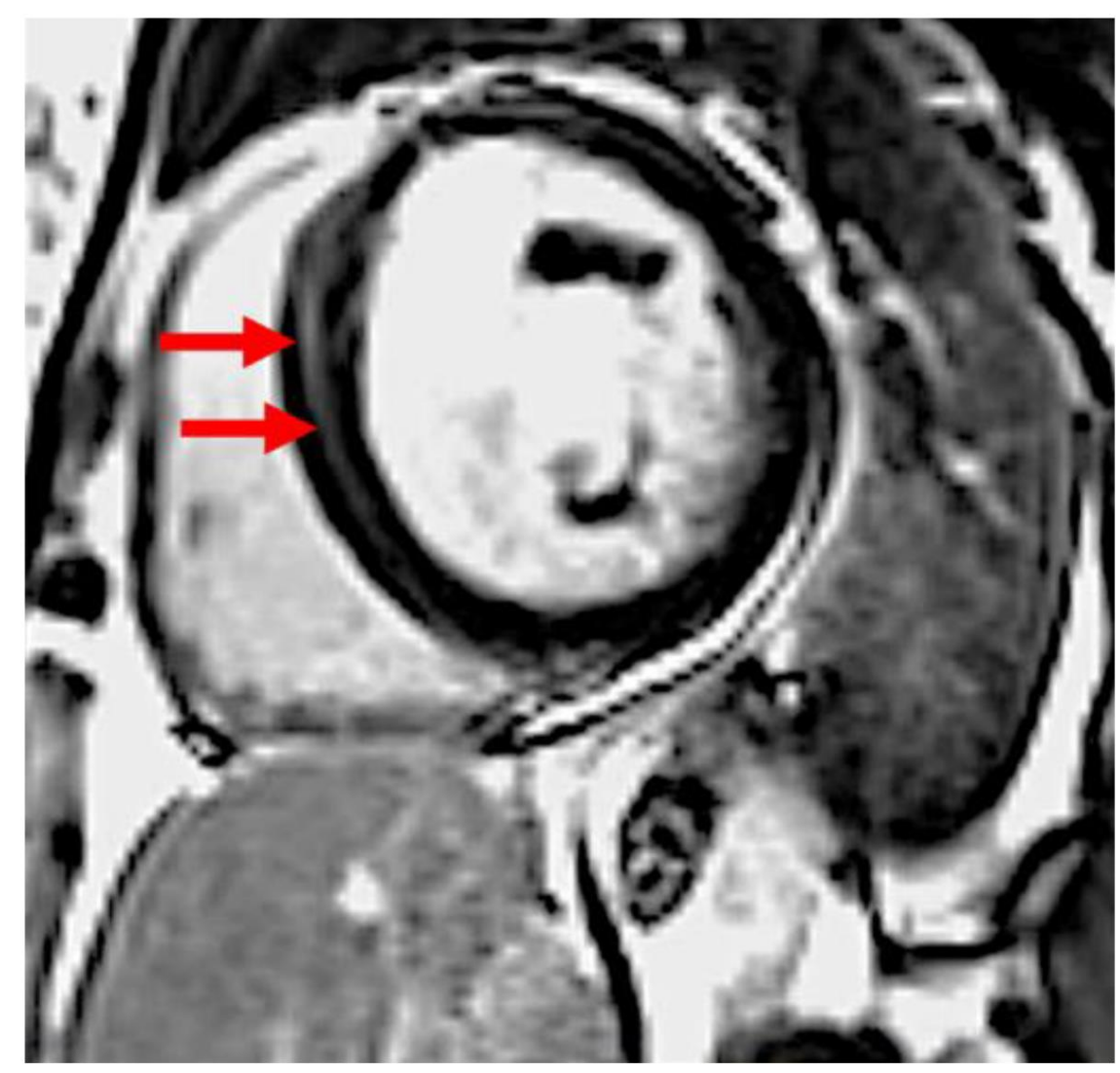

Figure 1. Late gadolinium enhancement in basal antero-septal wall in a patient with peripartum cardiomyopathy. Arrows indicate abnormal signal in the septum.

\subsection{Image Analysis}

SSFP images were used to calculate left (LV) and right ventricular (RV) volumes and ejection fractions (EF) with the use of dedicated software (Syngo via. Siemens, Erlangen, Germany), as previously described [14]. Left atrial (LA) and right atrial (RA) volumetric and functional calculations were performed separately by two independent and skilled observers (JPM and LM) according to a previously described protocol [15].

\subsection{Feature Tracking}

CMR-FT analysis was carried out by using CVi42 (Circle Cardiovascular Imaging Inc, Calgary, AB, Canada). LV and RV end-diastolic endocardial and LV epicardial contours were drawn automatically. RV epicardial contours were manually traced at the end-diastole. All contours were propagated, with manual readjustments performed as required. The global longitudinal strain and global longitudinal strain rate parameters were obtained from the 4-chamber view for the RV (RVGLS and RVGLS rate, respectively) and from the 2-chamber and 4-chamber views for the LV (LVGLS and LVGLS rate, respectively). Circumferential and radial strains and strain rates parameters for both the RV (RVCR, RVCR rate, RVR, and RVR rate) and the LV (LVCR, LVCR rate, LVR, and LVR rate) were determined in the short-axis view in the basal and mid-sections of the ventricles. Due to the known tendency for artifacts and lower muscle thickness in periapical slices, these images were not included in the FT analysis. The basal slice in the short-axis view was defined as the first slice below the atrioventricular level showing a circumferential myocardial enclosing. The midventricular slice was localized at the level of both papillary muscles. The values of the circumferential and radial strains and strain rates obtained in each slice of RV and LV were averaged.

The atrial strain and strain rates were also analyzed (Figure 2). LA endocardial borders in the two-chamber and four-chamber views, and RA borders in the four-chamber 
view were automatically drawn and propagated, with manual readjustments performed as required. Three aspects of LA and RA mechanics were analyzed: passive global longitudinal strain (GLS passive), active global longitudinal strain (GLS active), and total global longitudinal strain, which is the sum of the passive and active strains (GLS total). Accordingly, three strain rate parameters were evaluated: the peak positive strain rate (SRS), the peak early negative strain rate (SRE), and the peak late negative strain rate (SRA).

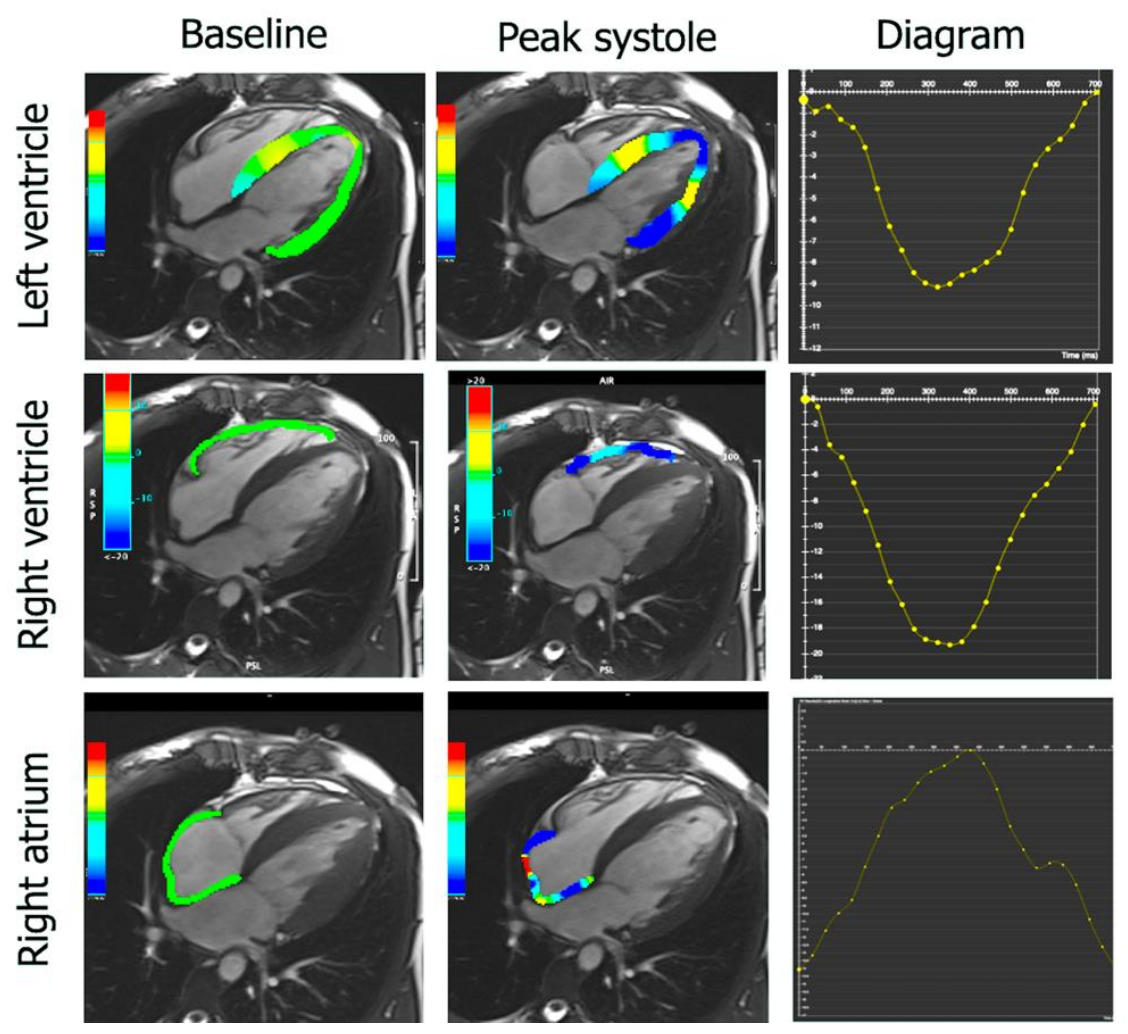

Figure 2. Longitudinal strain analysis in left and right ventricles and in the right atrium. Cardiovascular magnetic resonance feature tracking with the use of CVi42 in a patient with peripartum cardiomyopathy.

\subsection{Statistics}

All continuous variables were expressed as the mean \pm standard deviation (SD) or as the median and interquartile range and were tested for normal distribution with the use of the Kolmogorov-Smirnov test. Comparisons between groups were performed using the Student's $t$-test or the Wilcoxon-Mann-Whitney U test for continuous variables, and chi-square or Fisher's exact tests were used for categorical variables where appropriate. Intraobserver and interobserver variability were evaluated by using the Bland-Altman test and expressed as the mean difference $\pm \mathrm{SD}$, intraclass correlation coefficients (ICCs) with a $95 \%$ confidence interval $(\mathrm{CI})$, and the coefficient of variation ( $\mathrm{CoV})$. A two-sided $p$ value of less than 0.05 was considered to indicate statistical significance. Statistical analyses were performed with MedCalc 12.1.4.0 software (MedCalc, Mariakerke, Belgium).

\section{Results}

Overall, we included 21 patients with PPCM and 30 female patients with a diagnosis of DCM, matched for ejection fraction ( $35.9 \pm 13.7 \%$ vs. $35.2 \pm 11.2 \%, p=n s)$. Expectedly, DCM patients were older than PPCM patients ( $41.5 \pm 16.8$ vs. $30.5 \pm 5.9$ years, $p=0.03$ ). PPCM patients more often presented with severe symptoms of heart failure as assessed by NYHA classification ( 2.97 vs. $2.20, p=0.001)$ and had higher resting heart rates $(83.1 \pm 15.9$ vs. $69.5 \pm 11.4$ beats $/ \mathrm{min}, p=0.002$ ) as they less frequently received $\beta$-blocker therapy in comparison to DCM patients ( $81 \%$ vs. $100 \%, p=0.02$ ) 
During follow up, among the 21 PPCM patients, one patient underwent heart transplantation, one patient required temporary left ventricular assist device implantation, and one patient underwent biventricular assist device implantation with subsequent explantation following clinical improvement. Apart from the three patients mentioned above, the left ventricular ejection fraction remained impaired (below 50\%) in another four patients with PPCM.

There were no differences in the left and right ventricular volumetric and functional parameters, apart from LV stroke volume index, which was higher in DCM than compared to PPCM $\left(50.3 \pm 11.8 \mathrm{~mL} / \mathrm{m}^{2}\right.$ vs. $\left.44.5 \pm 10.5 \mathrm{~mL} / \mathrm{m}^{2}, p=0.04\right)$. Similarly, there were no differences in left atrial volumetric and functional parameters. However, in patients with PPCM, right atrial minimal and pre-systolic volumes were higher than in women with $\operatorname{DCM}(47.2 \pm 16.0 \mathrm{~mL}$ vs. $37.2 \pm 8.2 \mathrm{~mL}, p<0.01$ and $68.7 \pm 18.3 \mathrm{~mL}$ vs. $58.4 \pm 13.9 \mathrm{~mL}$, $p=0.02$, respectively). Moreover, the total RA EF was lower in PPCM in comparison to $\operatorname{DCM}(42.4 \pm 10.0 \mathrm{~mL}$ vs. $48.6 \pm 9.8 \mathrm{~mL}, p=0.02)$. There were no differences with regards to the frequency and the extent of LGE between PPCM patients and women with DCM (Table 1).

Table 1. Volumetric and functional CMR characteristics between patients with PPCM and female patients with DCM.

\begin{tabular}{|c|c|c|c|}
\hline & $\begin{array}{l}\text { PPCM } \\
\mathrm{n}=21\end{array}$ & $\begin{array}{c}\text { Female DCM } \\
\quad n=30\end{array}$ & $p$ \\
\hline Age (years) & $30.5 \pm 5.9$ & $41.5 \pm 16.8$ & 0.03 \\
\hline $\operatorname{BSA}\left(\mathrm{m}^{2}\right)$ & $1.76 \pm 0.19$ & $1.77 \pm 0.16$ & 0.91 \\
\hline Diabetes n (\%) & $0(0 \%)$ & $2(7 \%)$ & 0.23 \\
\hline Arterial hypertension (\%) & $3(14 \%)$ & $2(7 \%)$ & 0.17 \\
\hline NYHA class & 2.97 & 2.20 & 0.001 \\
\hline HR (beats/min) & $83.1 \pm 15.9$ & $69.5 \pm 11.4$ & 0.002 \\
\hline SBP (mmHg) & $107.3 \pm 19.2$ & $110.9 \pm 14.1$ & 0.47 \\
\hline $\mathrm{DBP}(\mathrm{mmHg})$ & $69.2 \pm 15.0$ & $68.9 \pm 8.9$ & 0.93 \\
\hline Troponin $\mathrm{T}(\mathrm{ng} / \mathrm{mL})$ & $10.7(0.001-6800)$ & $5.45(0.001-23.7)$ & 0.34 \\
\hline NT-proBNP (pg/mL) & $3007(27-35,000)$ & $2167(79-3036)$ & 0.048 \\
\hline B-blocker n (\%) & $17(81 \%)$ & $30(100 \%)$ & 0.02 \\
\hline ACE-I/ARB n (\%) & $20(95 \%)$ & $30(100 \%)$ & 0.16 \\
\hline $\begin{array}{l}\text { Aldosterone antagonist } \\
\mathrm{n}(\%)\end{array}$ & $15(71 \%)$ & $20(66 \%)$ & 0.39 \\
\hline \multicolumn{4}{|c|}{ Left ventricle } \\
\hline LVEDV (mL) & $245.5 \pm 97.4$ & $266.9 \pm 61.6$ & 0.36 \\
\hline LVESV (mL) & $167.1 \pm 89.7$ & $178.2 \pm 68.6$ & 0.63 \\
\hline LVSV (mL) & $78.6 \pm 22.0$ & $88.5 \pm 20.3$ & 0.12 \\
\hline LVEF (\%) & $35.9 \pm 13.7$ & $35.2 \pm 11.2$ & 0.85 \\
\hline $\operatorname{LVM}(\mathrm{g})$ & $125.9 \pm 33.2$ & $126.1 \pm 36.2$ & 0.99 \\
\hline $\operatorname{LVEDVI}\left(\mathrm{mL} / \mathrm{m}^{2}\right)$ & $138.3 \pm 50.1$ & $151.7 \pm 35.5$ & 0.29 \\
\hline LVESVI $\left(\mathrm{mL} / \mathrm{m}^{2}\right)$ & $93.7 \pm 48.6$ & $101.3 \pm 39.1$ & 0.56 \\
\hline LVSVI $\left(\mathrm{mL} / \mathrm{m}^{2}\right)$ & $44.5 \pm 10.5$ & $50.3 \pm 11.8$ & 0.04 \\
\hline LVMI $\left(\mathrm{g} / \mathrm{m}^{2}\right)$ & $72.2 \pm 16.8$ & $71.2 \pm 19.7$ & 0.86 \\
\hline
\end{tabular}


Table 1. Cont.

\begin{tabular}{|c|c|c|c|}
\hline & $\begin{array}{c}\text { PPCM } \\
n=21\end{array}$ & $\begin{array}{c}\text { Female DCM } \\
\quad n=30\end{array}$ & $p$ \\
\hline \multicolumn{4}{|c|}{ Right ventricle } \\
\hline RVEDV (mL) & $162.9 \pm 44.7$ & $152.1 \pm 39.8$ & 0.39 \\
\hline RVESV (mL) & $96.2 \pm 43.8$ & $93.2 \pm 45.1$ & 0.82 \\
\hline RVSV (mL) & $66.8 \pm 23.4$ & $59.1 \pm 20.2$ & 0.24 \\
\hline RVEF (\%) & $42.6 \pm 13.2$ & $41.1 \pm 14.3$ & 0.74 \\
\hline $\operatorname{RVEDVI}\left(\mathrm{mL} / \mathrm{m}^{2}\right)$ & $92.7 \pm 23.4$ & $85.9 \pm 23.0$ & 0.33 \\
\hline RVESVI $\left(\mathrm{mL} / \mathrm{m}^{2}\right)$ & $54.8 \pm 25.3$ & $52.0 \pm 23.4$ & 0.70 \\
\hline $\operatorname{RVSVI}\left(\mathrm{mL} / \mathrm{m}^{2}\right)$ & $37.6 \pm 11.5$ & $34.1 \pm 13.6$ & 0.37 \\
\hline \multicolumn{4}{|c|}{ Left atrium } \\
\hline LA area $\left(\mathrm{cm}^{2}\right)$ & $26.1 \pm 7.5$ & $27.2 \pm 5.4$ & 0.57 \\
\hline LAV max (mL) & $93.1 \pm 34.7$ & $93.9 \pm 21.2$ & 0.93 \\
\hline $\mathrm{LAV} \min (\mathrm{mL})$ & $50.2 \pm 28.5$ & $46.2 \pm 19.8$ & 0.61 \\
\hline LAV pac (mL) & $74.6 \pm 35.8$ & $73.5 \pm 24.2$ & 0.91 \\
\hline Total LAEF (\%) & $48.9 \pm 13.6$ & $52.3 \pm 12.1$ & 0.41 \\
\hline Passive LAEF (\%) & $23.0 \pm 12.0$ & $23.0 \pm 12.1$ & 0.99 \\
\hline Active LAEF (\%) & $33.9 \pm 12.6$ & $37.8 \pm 14.6$ & 0.40 \\
\hline \multicolumn{4}{|c|}{ Right atrium } \\
\hline RA area & $22.3 \pm 4.2$ & $19.8 \pm 3.6$ & 0.01 \\
\hline $\mathrm{RAV} \max (\mathrm{mL})$ & $82.0 \pm 22.0$ & $74.3 \pm 24.2$ & 0.26 \\
\hline $\mathrm{RAV} \min (\mathrm{mL})$ & $47.2 \pm 16.0$ & $37.2 \pm 8.2$ & $<0.01$ \\
\hline RAV pac (mL) & $68.7 \pm 18.3$ & $58.4 \pm 13.9$ & 0.02 \\
\hline Total RAEF (\%) & $42.4 \pm 10.0$ & $48.6 \pm 9.8$ & 0.02 \\
\hline Passive RAEF (\%) & $15.6 \pm 8.7$ & $20.0 \pm 12.5$ & 0.24 \\
\hline Active RAEF (\%) & $31.7 \pm 10.3$ & $35.6 \pm 8.0$ & 0.19 \\
\hline \multicolumn{4}{|c|}{ LGE } \\
\hline LGE n $(\%)$ & $9(43 \%)$ & $18(60 \%)$ & 0.22 \\
\hline LGE \% (IQR) & $1.7 \%(0-2.9 \%)$ & $2.3 \%(0-4.5 \%)$ & 0.63 \\
\hline
\end{tabular}

ACE-I—angiotensin converting enzyme inhibitor; ARB—angiotensin receptor blocker; BSA—body surface area; CMR — cardiovascular magnetic resonance; DBP—diastolic blood pressure; HR - heart rate; LA-left atrium; LAEF—left atrial ejection fraction; LAV max-left atrial maximal volume; LAV min—left atrial minimal volume; LAV pac—left atrial pre-systolic volume; LVEDV—left ventricular end diastolic volume; LVEDVI—left ventricular end diastolic volume index; LVEF—left ventricular ejection fraction; LVESV—left ventricular end systolic volume; LVESVI-left ventricular end systolic volume index; LGE-late gadolinium enhancement; LVM-left ventricular myocardial mass; LVSV—-left ventricular stroke volume; LVMI - left ventricular myocardial mass; LVSVI—left ventricular stroke volume index; NT-proBNP-N-terminal pro b-type natriuretic peptide; NYHA-New York Heart Association heart failure class; RA—right atrium; RAEF—right atrial ejection fraction; RAV max —right atrial maximal volume; RAV min—right atrial minimal volume; RAV pac-right atrial pre-systolic volume; RVEDV—right ventricular end diastolic volume; RVEDVI—right ventricular end diastolic volume index; RVESV— right ventricular end systolic volume; RVESVI—right ventricular end systolic volume index; RVEF—right ventricular ejection fraction; RVSV—right ventricular stroke volume; RVSVI—right ventricular stroke volume index; SBP—systolic blood pressure.

The distribution of LGE was similar in both groups. In the PPCM group, most of patients ( $\mathrm{n}=8,89 \%$ ) presented with mid-wall fibrosis of the interventricular septum and/or the inferior insertion point. Only one PPCM patient was found to have subendocardial LGE on CMR. Similarly, mid-wall fibrosis was detected in 10 (55\%) female DCM patients. 
In the remaining eight (45\%) DCM patients, LGE of both mid-wall and subendocardial distribution was observed.

Strain analysis showed that patients with PPCM had less impaired left ventricular GLS $(-9.6 \pm 3.9$ vs. $-7.7 \pm 2.6, p=0.03)$ and GLS rates $(-0.55 \pm 0.14$ vs. $-0.45 \pm 0.12$, $p<0.01)$ in comparison with female patients with DCM. Furthermore, PPCM patients had less impaired left ventricular GCS rates $(-0.64 \pm 0.26$ vs. $-0.53 \pm 0.13, p=0.04)$. However, there were no differences in the right ventricular and biatrial mechanical indices between the groups (Table 2).

Table 2. CMR-FT derived strain parameters in patients with PPCM and female patients with DCM.

\begin{tabular}{|c|c|c|c|}
\hline & $\begin{array}{l}\text { PPCM } \\
n=21\end{array}$ & $\begin{array}{c}\text { Female DCM } \\
n=30\end{array}$ & $p$ \\
\hline \multicolumn{4}{|c|}{ Left ventricle } \\
\hline LV GRS (\%) & $14.1 \pm 6.4$ & $13.9 \pm 4.9$ & 0.90 \\
\hline LV GCS (\%) & $-11.0 \pm 4.6$ & $-9.8 \pm 3.3$ & 0.32 \\
\hline LV GLS (\%) & $-9.6 \pm 3.9$ & $-7.7 \pm 2.6$ & 0.03 \\
\hline LV GRS rate $\left(\mathrm{s}^{-1}\right)$ & $0.91 \pm 0.44$ & $0.76 \pm 0.21$ & 0.22 \\
\hline LV GCS rate $\left(\mathrm{s}^{-1}\right)$ & $-0.64 \pm 0.26$ & $-0.53 \pm 0.13$ & 0.04 \\
\hline LV GLS rate $\left(\mathrm{s}^{-1}\right)$ & $-0.55 \pm 0.14$ & $-0.45 \pm 0.12$ & $<0.01$ \\
\hline \multicolumn{4}{|c|}{ Right ventricle } \\
\hline RV GRS (\%) & $14.1 \pm 6.2$ & $13.2 \pm 8.9$ & 0.70 \\
\hline RV GCS (\%) & $-9.1 \pm 3.8$ & $-8.3 \pm 6.4$ & 0.62 \\
\hline RV GLS (\%) & $-14.7 \pm 7.1$ & $13.8 \pm 6.8$ & 0.68 \\
\hline RV GRS rate $\left(\mathrm{s}^{-1}\right)$ & $1.01 \pm 0.49$ & $0.83 \pm 0.47$ & 0.21 \\
\hline RV GCS rate $\left(\mathrm{s}^{-1}\right)$ & $-0.54 \pm 0.28$ & $-0.48 \pm 0.26$ & 0.49 \\
\hline RV GLS rate $\left(\mathrm{s}^{-1}\right)$ & $-1.01 \pm 0.40$ & $-0.86 \pm 0.31$ & 0.20 \\
\hline \multicolumn{4}{|c|}{ Left atrium } \\
\hline LA GLS total (\%) & $17.5 \pm 9.2$ & $19.8 \pm 9.1$ & 0.43 \\
\hline LA GLS passive (\%) & $9.3 \pm 5.1$ & $10.4 \pm 4.1$ & 0.46 \\
\hline LA GLS active (\%) & $8.2 \pm 6.3$ & $8.7 \pm 4.4$ & 0.74 \\
\hline LA SRS $\left(\mathrm{s}^{-1}\right)$ & $0.72 \pm 0.35$ & $0.75 \pm 0.40$ & 0.80 \\
\hline LA SRE $\left(s^{-1}\right)$ & $-0.66 \pm 0.41$ & $-0.83 \pm 0.50$ & 0.25 \\
\hline LA SRA $\left(\mathrm{s}^{-1}\right)$ & $-0.70 \pm 0.40$ & $-0.62 \pm 0.36$ & 0.57 \\
\hline \multicolumn{4}{|c|}{ Right atrium } \\
\hline RA GLS total (\%) & $26.6 \pm 9.6$ & $26.5 \pm 9.1$ & 0.96 \\
\hline RA GLS passive (\%) & $11.2 \pm 6.3$ & $11.4 \pm 5.8$ & 0.94 \\
\hline RA GLS active (\%) & $15.4 \pm 8.1$ & $13.2 \pm 5.8$ & 0.32 \\
\hline RA SRS $\left(s^{-1}\right)$ & $1.18 \pm 0.40$ & $1.14 \pm 0.42$ & 0.80 \\
\hline RA SRE $\left(\mathrm{s}^{-1}\right)$ & $-0.59 \pm 0.32$ & $-0.72 \pm 0.48$ & 0.34 \\
\hline RA SRA $\left(s^{-1}\right)$ & $-1.00 \pm 0.67$ & $-0.89 \pm 0.39$ & 0.58 \\
\hline
\end{tabular}

GCS—global circumferential strain; GLS—global longitudinal strain; GRS—global radial strain, LA—left atrium; LV—left ventricle; RA—right atrium; RV—right ventricle; SRA—late negative strain rate; SRE—early negative strain rate; SRS- peak positive strain rate.

Expectedly, both PPCM and DCM patients with LGE had more dilated left ventricles and more impaired LV function in comparison with PPCM and DCM patients without LGE (Table 3). Additionally, female DCM patients with fibrosis detected by CMR had higher 
LV mass $(p=0.04)$ and LV mass index $(p<0.01)$ in comparison to DCM patients without fibrosis.

Table 3. Volumetric and functional CMR characteristics between female patients with PPCM and DCM with and without LGE.

\begin{tabular}{|c|c|c|c|c|c|c|}
\hline & $\begin{array}{l}\text { PPCM without LGE } \\
n=12(67 \%)\end{array}$ & $\begin{array}{l}\text { PPCM with LGE } \\
\text { n }=9(43 \%)\end{array}$ & $p$ & $\begin{array}{l}\text { DCM without LGE } \\
n=12(40 \%)\end{array}$ & $\begin{array}{c}\text { DCM with LGE } \\
n=18(60 \%)\end{array}$ & $p$ \\
\hline Age (years) & $29.9 \pm 6.3$ & $31.4 \pm 5.7$ & $\mathrm{~ns}$ & $44.4 \pm 14.8$ & $39.6 \pm 18.6$ & 0.44 \\
\hline \multicolumn{7}{|c|}{ Left ventricle } \\
\hline LVEDV (mL) & $201.9 \pm 81.1$ & $307.7 \pm 88.1$ & 0.022 & $236.0 \pm 63.3$ & $287.4 \pm 54.3$ & 0.02 \\
\hline LVESV (mL) & $122.6 \pm 70.0$ & $230.6 \pm 78.2$ & 0.009 & $141.5 \pm 56.9$ & $202.7 \pm 67.2$ & 0.01 \\
\hline LVSV (mL) & $79.5 \pm 25.6$ & $77.3 \pm 17.5$ & 0.84 & $94.3 \pm 15.8$ & $84.7 \pm 22.9$ & 0.20 \\
\hline $\operatorname{LVEF}(\%)$ & $42.5 \pm 13.0$ & $26.4 \pm 8.1$ & 0.007 & $41.2 \pm 8.3$ & $31.2 \pm 11.4$ & $<0.01$ \\
\hline $\operatorname{LVM}(\mathrm{g})$ & $121.3 \pm 38.3$ & $132.4 \pm 25.6$ & 0.51 & $110.7 \pm 31.9$ & $136.3 \pm 36.9$ & 0.04 \\
\hline LVEDVI (mL/m²) & $114.7 \pm 39.8$ & $172.0 \pm 45.5$ & 0.015 & $129.5 \pm 30.5$ & $166.6 \pm 31.8$ & $<0.01$ \\
\hline $\operatorname{LVESVI}\left(\mathrm{mL} / \mathrm{m}^{2}\right)$ & $69.2 \pm 37.1$ & $128.7 \pm 42.3$ & 0.008 & $77.3 \pm 28.1$ & $117.3 \pm 38.1$ & $<0.01$ \\
\hline $\operatorname{LVSVI}\left(\mathrm{mL} / \mathrm{m}^{2}\right)$ & $45.3 \pm 11.9$ & $43.3 \pm 8.8$ & 0.71 & $52.2 \pm 9.1$ & $49.1 \pm 13.6$ & 0.49 \\
\hline $\operatorname{LVMI}\left(\mathrm{g} / \mathrm{m}^{2}\right)$ & $69.2 \pm 17.6$ & $76.4 \pm 15.7$ & 0.40 & $60.3 \pm 13.8$ & $78.4 \pm 20.2$ & $<0.01$ \\
\hline \multicolumn{7}{|c|}{ Right ventricle } \\
\hline RVEDV (mL) & $146.9 \pm 45.1$ & $185.7 \pm 35.2$ & 0.08 & $134.8 \pm 41.7$ & $163.6 \pm 36.2$ & 0.04 \\
\hline RVESV (mL) & $75.0 \pm 27.9$ & $126.4 \pm 46.2$ & 0.012 & $70.3 \pm 35.1$ & $108.4 \pm 46.2$ & 0.01 \\
\hline RVSV (mL) & $72.0 \pm 25.5$ & $59.4 \pm 19.5$ & 0.29 & $64.5 \pm 21.3$ & $55.4 \pm 20.3$ & 0.23 \\
\hline RVEF (\%) & $49.1 \pm 8.8$ & $33.4 \pm 13.5$ & 0.011 & $49.2 \pm 13.7$ & $35.8 \pm 16.3$ & 0.02 \\
\hline RVEDVI (mL/m²) & $83.8 \pm 22.9$ & $105.4 \pm 18.7$ & 0.057 & $74.0 \pm 23.5$ & $93.9 \pm 20.1$ & 0.02 \\
\hline $\operatorname{RVESVI}\left(\mathrm{mL} / \mathrm{m}^{2}\right)$ & $42.7 \pm 16.6$ & $72.0 \pm 26.5$ & 0.013 & $38.3 \pm 18.0$ & $61.1 \pm 22.9$ & $<0.01$ \\
\hline $\operatorname{RVSVI}\left(\mathrm{mL} / \mathrm{m}^{2}\right)$ & $40.4 \pm 12.3$ & $33.6 \pm 5.4$ & 0.24 & $35.8 \pm 14.1$ & $32.9 \pm 14.1$ & 0.56 \\
\hline \multicolumn{7}{|c|}{ Left atrium } \\
\hline LA area $\left(\mathrm{cm}^{2}\right)$ & $21.4 \pm 4.7$ & $32.7 \pm 5.4$ & 0.0003 & $26.5 \pm 5.3$ & $27.7 \pm 5.8$ & 0.57 \\
\hline $\mathrm{LAV} \max (\mathrm{mL})$ & $73.5 \pm 28.2$ & $115.6 \pm 29.8$ & 0.015 & $90.6 \pm 17.8$ & $96.1 \pm 24.6$ & 0.53 \\
\hline LAV $\min (\mathrm{mL})$ & $30.7 \pm 14.5$ & $72.4 \pm 25.1$ & 0.001 & $37.8 \pm 16.7$ & $51.5 \pm 21.4$ & 0.09 \\
\hline LAV pac (mL) & $51.5 \pm 23.7$ & $101.1 \pm 30.2$ & 0.004 & $68.0 \pm 24.4$ & $77.0 \pm 25.9$ & 0.37 \\
\hline Total LAEF (\%) & $58.3 \pm 10.1$ & $38.1 \pm 8.6$ & 0.001 & $59.3 \pm 11.7$ & $47.9 \pm 11.3$ & 0.02 \\
\hline Passive LAEF (\%) & $31.3 \pm 8.6$ & $13.5 \pm 8.2$ & 0.001 & $26.6 \pm 14.4$ & $20.6 \pm 12.3$ & 0.25 \\
\hline Active LAEF (\%) & $39.0 \pm 14.2$ & $28.2 \pm 8.8$ & 0.11 & $44.4 \pm 12.8$ & $33.7 \pm 15.5$ & 0.07 \\
\hline
\end{tabular}


Table 3. Cont.

\begin{tabular}{|c|c|c|c|c|c|c|}
\hline & $\begin{array}{l}\text { PPCM without LGE } \\
\text { n = } 12(67 \%)\end{array}$ & $\begin{array}{c}\text { PPCM with LGE } \\
n=9(43 \%)\end{array}$ & $p$ & $\begin{array}{c}\text { DCM without LGE } \\
n=12(40 \%)\end{array}$ & $\begin{array}{c}\text { DCM with LGE } \\
n=18(60 \%)\end{array}$ & $p$ \\
\hline \multicolumn{7}{|c|}{ Right atrium } \\
\hline RA area & $21.7 \pm 4.9$ & $23.3 \pm 3.1$ & 0.46 & $22.2 \pm 3.1$ & $18.0 \pm 2.9$ & $<0.01$ \\
\hline $\mathrm{RAV} \max (\mathrm{mL})$ & $84.0 \pm 26.3$ & $79.7 \pm 18.8$ & 0.73 & $82.1 \pm 16.9$ & $69.4 \pm 21.6$ & 0.11 \\
\hline $\mathrm{RAV} \min (\mathrm{mL})$ & $48.0 \pm 16.9$ & $46.3 \pm 16.8$ & 0.84 & $40.0 \pm 5.9$ & $35.5 \pm 9.6$ & 0.18 \\
\hline RAV pac (mL) & $67.1 \pm 19.1$ & $70.6 \pm 19.6$ & 0.73 & $61.0 \pm 14.9$ & $56.7 \pm 14.5$ & 0.49 \\
\hline Total RAEF (\%) & $42.1 \pm 11.1$ & $42.8 \pm 9.8$ & 0.91 & $50.3 \pm 9.3$ & $47.5 \pm 10.8$ & 0.01 \\
\hline Passive RAEF (\%) & $19.2 \pm 5.8$ & $11.4 \pm 10.4$ & 0.09 & $25.0 \pm 15.1$ & $16.8 \pm 11.0$ & 0.01 \\
\hline Active RAEF (\%) & $28.7 \pm 11.0$ & $35.2 \pm 9.3$ & 0.24 & $33.3 \pm 6.4$ & $37.0 \pm 9.2$ & 0.46 \\
\hline \multicolumn{7}{|c|}{$\begin{array}{l}\text { BSA-body surface area; CMR_cardiovascular magnetic resonance; LA-left atrium; LAEF-left atrial ejection fraction; LAV max-left } \\
\text { atrial maximal volume; LAV min-left atrial minimal volume; LAV pac-left atrial pre-systolic volume; LVEDV-left ventricular end } \\
\text { diastolic volume; LVEDVI-left ventricular end diastolic volume index; LVEF-left ventricular ejection fraction; LVESV-left ventricular } \\
\text { end systolic volume; LVESVI-left ventricular end systolic volume index; LGE-late gadolinium enhancement; LVM-left ventricular } \\
\text { myocardial mass; LVSV-left ventricular stroke volume; LVMI-left ventricular myocardial mass; LVSVI-left ventricular stroke volume } \\
\text { index; RA-right atrium; RAEF—right atrial ejection fraction; RAV max-right atrial maximal volume; RAV min-right atrial minimal } \\
\text { volume; RAV pac-right atrial pre-systolic volume; RVEDV-right ventricular end diastolic volume; RVEDVI-right ventricular end } \\
\text { diastolic volume index; RVESV-right ventricular end systolic volume; RVESVI-right ventricular end systolic volume index; RVEF-right } \\
\text { ventricular ejection fraction; RVSV-right ventricular stroke volume; RVSVI-right ventricular stroke volume index. }\end{array}$} \\
\hline
\end{tabular}

Regarding the RV, PPCM and DCM patients with LGE had higher RV end-systolic volume (PPCM: $p=0.01$; DCM: $p=0.01$ ), RV end-systolic volume index (PPCM: $p=0.01$; DCM: $p<0.01$ ), and more impaired RV EF (PPCM: $p=0.01$; DCM: $p=0.02$ ). Additionally, female DCM patients with LGE also had higher RV end-diastolic volume $(p=0.04)$ and RV end-diastolic volume index $(p=0.02)$ when compared to DCM women without LGE.

Furthermore, PPCM patients with LGE on CMR had larger LA (area: $p<0.01$; LAV max: $p=0.01$; LAV min: $p<0.01$; LAV pre-ac: $p<0.01)$ and more impaired total $(p<0.01)$ and passive $(p<0.01)$ LA EF than PPCM patients without LGE. Female patients with DCM presenting with LGE had lower total LA EF $(p=0.02)$ than compared to women without LGE. While there were no differences in RA volumetric and functional indices in the PPCM group, DCM patients with LGE had smaller right atria $(p<0.01)$ and lower total $(p=0.01)$ and passive $(p=0.01)$ RA EF in comparison with DCM patients without LGE.

The analysis of all left ventricular strain and strain rate parameters showed that they were more impaired in patients with LGE than in patients without LGE in both PPCM (GLS: $p<0.01$; GCS: $p=0.01$; GRS: $p<0.01$; GCS rate: $p=0.01$; GRS rate: $p=0.01$ ) and DCM groups (GLS: $p=0.01$; GCS: $p<0.01$; GRS: $p<0.01$; GCS rate: $p<0.01$; GRS rate: $p=0.04$ ). In PPCM group the mean value of LV GLS rate was higher in patients without LGE than in LGE positive patients but the difference did not meet statistical significance $(p=0.053)$.

With regards to RV mechanics, PPCM and DCM patients with LGE had more impaired RV GCS (PPCM: $p=0.01$; DCM: $p=0.01$ ), RV GRS (PPCM: $p<0.01$; DCM: $p=0.03$ ), and GRS rate (PPCM: $p=0.04$; DCM: $p=0.01$ ) in comparison to PPCM and DCM patients without LGE. Additionally, female DCM patients with LGE had more impaired GCS rate in comparison with DCM patients without LGE $(-0.39 \pm 0.27$ vs. $-0.62 \pm 0.19, p=0.01)$.

Similarly, some of the LA mechanical indices were better preserved in PPCM (LA GLS total: $p<0.01$; LA GLS active: $p<0.01$; LA SRS: $p=0.01$ ) and DCM (LA GLS total: $p<0.01$; LA GLS active: $p<0.01$ ) patients without LGE than in PPCM and DCM patients with LGE. There were no differences in the right atrial mechanical indices in PPCM patients with and without LGE. While in DCM patients with fibrosis, the RA GLS total $(p=0.01)$, RA GLS passive $(p<0.01)$, and RA SRS $(p=0.02)$ were more impaired than in the group of DCM patients without LGE (Table 4). 
Table 4. CMR-FT derived strain parameters in patients with PPCM with and without LGE.

\begin{tabular}{|c|c|c|c|c|c|c|}
\hline & $\begin{array}{l}\text { PPCM without LGE } \\
n=12(67 \%)\end{array}$ & $\begin{array}{l}\text { PPCM with LGE } \\
n=9(43 \%)\end{array}$ & $p$ & $\begin{array}{l}\text { DCM without LGE } \\
n=12(40 \%)\end{array}$ & $\begin{array}{c}\text { DCM with LGE } \\
n=18(60 \%)\end{array}$ & $p$ \\
\hline \multicolumn{7}{|c|}{ Left ventricle } \\
\hline LV GRS (\%) & $17.9 \pm 5.8$ & $9.2 \pm 3.4$ & 0.002 & $16.9 \pm 2.7$ & $11.9 \pm 5.2$ & $<0.01$ \\
\hline LV GCS (\%) & $-13.3 \pm 4.5$ & $-8.0 \pm 2.9$ & 0.013 & $-11.9 \pm 2.0$ & $-8.5 \pm 3.5$ & $<0.01$ \\
\hline LV GLS (\%) & $-12.3 \pm 3.4$ & $-6.6 \pm 1.9$ & 0.002 & $-9.1 \pm 1.7$ & $-6.8 \pm 2.8$ & 0.01 \\
\hline LV GRS rate $\left(\mathrm{s}^{-1}\right)$ & $1.1 \pm 0.43$ & $0.62 \pm 0.28$ & 0.015 & $0.86 \pm 0.21$ & $0.70 \pm 0.21$ & 0.04 \\
\hline LV GCS rate $\left(\mathrm{s}^{-1}\right)$ & $-0.78 \pm 0.22$ & $-0.46 \pm 0.21$ & 0.011 & $-0.62 \pm 0.11$ & $-0.47 \pm 0.13$ & $<0.01$ \\
\hline LV GLS rate $\left(\mathrm{s}^{-1}\right)$ & $-0.61 \pm 0.16$ & $-0.47 \pm 0.04$ & 0.053 & $-0.42 \pm 0.09$ & $-0.46 \pm 0.14$ & 0.38 \\
\hline \multicolumn{7}{|c|}{ Right ventricle } \\
\hline RV GRS (\%) & $17.9 \pm 5.3$ & $9.3 \pm 3.6$ & 0.002 & $17.1 \pm 6.2$ & $10.6 \pm 10.0$ & 0.03 \\
\hline RV GCS (\%) & $-11.1 \pm 2.3$ & $-6.5 \pm 3.3$ & 0.015 & $-11.7 \pm 5.3$ & $-6.1 \pm 6.6$ & 0.01 \\
\hline RV GLS (\%) & $-17.9 \pm 7.3$ & $-11.0 \pm 5.5$ & 0.06 & $-16.6 \pm 6.7$ & $-12.0 \pm 7.0$ & 0.09 \\
\hline RV GRS rate $\left(\mathrm{s}^{-1}\right)$ & $1.2 \pm 0.52$ & $0.74 \pm 0.36$ & 0.044 & $1.10 \pm 0.48$ & $0.64 \pm 0.40$ & 0.01 \\
\hline RV GCS rate $\left(\mathrm{s}^{-1}\right)$ & $-0.62 \pm 0.3$ & $-0.44 \pm 0.23$ & 0.21 & $-0.62 \pm 0.19$ & $-0.39 \pm 0.27$ & 0.01 \\
\hline RV GLS rate $\left(\mathrm{s}^{-1}\right)$ & $-1.09 \pm 0.42$ & $-0.92 \pm 0.41$ & 0.45 & $-1.01 \pm 0.32$ & $-0.78 \pm-0.30$ & 0.06 \\
\hline \multicolumn{7}{|c|}{ Left atrium } \\
\hline LA GLS total (\%) & $22.7 \pm 7.9$ & $10.8 \pm 6.6$ & 0.006 & $26.3 \pm 7.2$ & $15.8 \pm 8.4$ & $<0.01$ \\
\hline LA GLS passive (\%) & $11.1 \pm 4.9$ & $7.0 \pm 4.8$ & 0.11 & $12.0 \pm 3.0$ & $9.4 \pm 4.7$ & 0.12 \\
\hline LA GLS active (\%) & $12.5 \pm 5.2$ & $2.7 \pm 2.3$ & $<0.001$ & $11.9 \pm 2.1$ & $6.8 \pm 4.6$ & $<0.01$ \\
\hline LA SRS $\left(\mathrm{s}^{-1}\right)$ & $0.91 \pm 0.33$ & $0.50 \pm 0.24$ & 0.014 & $0.94 \pm 0.45$ & $0.64 \pm 0.35$ & 0.06 \\
\hline LA SRE $\left(\mathrm{s}^{-1}\right)$ & $-0.83 \pm-0.43$ & $-0.45 \pm 0.30$ & 0.06 & $-0.92 \pm 0.63$ & $-0.78 \pm 0.45$ & 0.50 \\
\hline LA SRA $\left(\mathrm{s}^{-1}\right)$ & $-0.72 \pm 0.46$ & $-0.66 \pm 0.37$ & 0.77 & $-0.74 \pm 0.37$ & $-0.56 \pm 0.37$ & 0.23 \\
\hline \multicolumn{7}{|c|}{ Right atrium } \\
\hline RA GLS total (\%) & $27.1 \pm 9.0$ & $26.0 \pm 11.4$ & 0.83 & $32.0 \pm 9.9$ & $23.1 \pm 7.5$ & 0.01 \\
\hline $\begin{array}{c}\text { RA GLS passive } \\
(\%)\end{array}$ & $10.5 \pm 5.3$ & $12.2 \pm 7.9$ & 0.62 & $15.1 \pm 6.6$ & $9.1 \pm 4.3$ & $<0.01$ \\
\hline RA GLS active (\%) & $16.7 \pm 10.0$ & $13.8 \pm 5.5$ & 0.51 & $11.9 \pm 4.0$ & $14.0 \pm 7.0$ & 0.37 \\
\hline RA SRS $\left(\mathrm{s}^{-1}\right)$ & $1.2 \pm 0.41$ & $1.1 \pm 0.42$ & 0.54 & $1.4 \pm 0.42$ & $0.99 \pm 0.39$ & 0.02 \\
\hline $\operatorname{RA} \operatorname{SRE}\left(\mathrm{s}^{-1}\right)$ & $-0.65 \pm 0.29$ & $-0.50 \pm 0.37$ & 0.39 & $-0.88 \pm 0.69$ & $-0.62 \pm 0.32$ & 0.25 \\
\hline RA SRA $\left(\mathrm{s}^{-1}\right)$ & $-0.79 \pm 0.49$ & $-1.2 \pm 0.85$ & 0.20 & $-0.79 \pm 0.42$ & $-0.96 \pm 0.41$ & 0.31 \\
\hline
\end{tabular}

GCS—global circumferential strain; GLS—global longitudinal strain; GRS—global radial strain; LA—left atrium; LV—left ventricle; RA—right atrium; RV—right ventricle; SRA—late negative strain rate; SRE—early negative strain rate; SRS—peak positive strain rate.

The indices of reproducibility for atrial and ventricular strain and strain rate parameters were satisfactory-intraobserver and interobserver ICCs ranged between 0.50 and 0.99 for all components of myocardial performance (Supplementary Materials, Table S1).

\section{Discussion}

Our main findings can be summarized as follows:

1. Patients with PPCM have lower LV stroke volume index and higher RA volume with lower RA total ejection fraction in comparison to women with DCM.

2. In patients with PPCM, some indices of LV mechanics such as LV GLS and LV GLS rate, as well as LV GRS rate, are less impaired than in DCM women. 
3. Both PPCM and DCM female patients with LGE have more dilated and impaired left and right ventricles in comparison to patients without LGE on CMR imaging.

4. Almost all LV and RV mechanical indices are more impaired in patients with LGE in comparison to patients without LGE in both PPCM and DCM groups.

\subsection{CMR for Diagnosis and Prognostication}

The role of CMR in the diagnosis and follow up process in patients with DCM and PPCM is well established. Firstly, an accurate assessment of RV size and function can be challenging by using other techniques, such as echocardiography, because of its complex and variable shape. Reduced RV ejection fraction on CMR is an independent predictor of all-cause mortality and adverse heart failure outcomes in DCM and PPCM [12,16]. In detail, an impaired RV is associated with a dilation of both ventricles and lower LVEF, suggesting more extensive biventricular cardiac involvement in PPCM [17]. CMR provides the gold standard noninvasive assessment of both RV and LV size and function because of its three-dimensional capabilities [18]. In our study, PPCM patients had more reduced LVSVI than DCM patients. This finding may be explained by the fact that myofilaments from PPCM patients show more impaired function than myofilaments from patients with DCM [1]. The reason for that may be the lack of expression of fetal EH-myomesin in PPCM. $\mathrm{EH}$-myomesin is responsible for stabilizing heart muscle function in DCM, but the presence of this remodeling disables DCM patients from total recovery that is observed in PPCM [1].

CMR also allows accurate quantification of left and right atrial volumes by using the biplane area-length method [19] and is superior than other noninvasive imaging methods because of its excellent endocardial border definition and multiplanar imaging ability, even in the presence of atrial fibrillation [20]. It has been proposed that the degree of LA enlargement acts as a barometer of diastolic dysfunction; therefore, the LA size is used to predict heart failure outcomes [21]. It has been previously demonstrated that indexed LA volume calculated by using CMR independently predicts cardiac transplant-free survival in DCM [22]. Interestingly, in our study, PPCM patients had larger right atrium, but not LA, with more impaired function in comparison to DCM patients. This finding may be associated with increased blood volume, as a physiological adaptation during pregnancy and peripartum period affects RA more than the LA volume. Furthermore, the impairment of RA function may reflect more severe diastolic dysfunction of overloaded RV. The LA was enlarged to a comparable degree in both PPCM and DCM patients.

Finally, given its accuracy in the assessment of cardiac muscle function, CMR is the method of choice for the follow up of patients with DCM after pharmacologic and surgical interventions [23] and should be applied to PPCM patients as well [9]. Given the favorable interobserver variability compared with other methods of assessment, the use of CMR can reduce the sample size required, reducing the overall cost and time needed to complete a research study [24]. In our study, the reproducibility of strain and strain-rate parameters was satisfactory, especially with regards to volumetric measurements.

\subsection{Fibrosis in PPCM}

Data on the role of LGE in PPCM are limited [25]. Importantly, fibrosis was detected histologically in samples obtained during heart transplantation from patients with PPCM [1]. In our study, LGE was noted in $43 \%$ patients with PPCM, which is similar to data reported by Arora et al., who demonstrated the presence of LGE in 40\% of PPCM patients on CMR [26]. It has been suggested that LGE in PPCM is time dependent, with LGE observed early in the course of the disease before LV function recovery [27]. Furthermore, other authors have reported the link between LGE in PPCM and an unfavorable prognosis with slower recovery, higher risk of prolonged or permanent systolic dysfunction, and higher rate of developing heart failure exacerbation in future pregnancies [28]. Likewise, the presence of fibrosis in DCM is known to be associated with adverse ventricular remodeling and increased all-cause mortality [29]. Therefore, the need for CMR with the assessment of fibrosis in the early course of PPCM, if possible, should be emphasized [9]. In PPCM, the 
degree of fibrosis may help advise on future pregnancies after recovery. Expectedly, in our study, PPCM patients with LGE, analogously to female DCM patients with LGE, presented with increased dilation of both ventricles along with impaired biventricular function in comparison to patients without LGE. A large prospective study of the prognostic value of fibrosis on CMR in PPCM is needed [30].

\subsection{Fibrosis and Myocardial Mechanics}

CMR-derived feature tracking strain analysis represents another promising tool for improving PPCM patients' risk stratification. There is evidence which suggests that FT parameters can predict survival in DCM and improve risk stratification beyond clinical parameters, biomarkers, LVEF, and LGE [31]. Similarly, in patients with PPCM, a study demonstrated that echocardiographic measures of GLS and GCS were additive to routine LV functional measures and clinical factors, such as black race, for identifying PPCM patients at risk [32]. Specifically, patients with more impaired LV global strain at presentation were at increased risk for impaired LV recovery, death, or LVAD implantation. Other results demonstrated that $L V$ longitudinal deformation is globally impaired in patients with PPCM, regardless of the LVEF values [33]. Our study showed that almost all LV and RV mechanical indices were more impaired in patients with fibrosis compared to patients without fibrosis in both the PPCM and DCM groups. The difference between PPCM patients with and without LGE with regards to LV GLS rate was at the limit of significance probably due to small number of patients studied and should be evaluated in further research.

In PPCM patients, LV GLS and LV GLS rates, as well as the LV GRS rate, were found to be less impaired than in women with DCM. This fact may be associated with shorter disease duration in the PPCM group despite the matched ejection fraction.

While there are data that echo-derived LVGLS was the most accurate method for detecting LV fibrosis as assessed by histopathologic examination in patients undergoing heart transplantation [34], this is the first study to link LGE with the severity of RV and LV deformation abnormalities in PPCM.

\subsection{Study Limitations}

The limitations of our study are related to its design. It is a single center retrospective study with a relatively small number of patients with PPCM. It must also be noted that CMR mapping techniques were not applied to our cohort, whereas they are now considered important in the diagnosis of PPCM. Our results may suggest the use of biventricular strain parameters for predicting fibrosis severity in PPCM [14]. However, due to the small sample size, we did not assess any association between strain values and outcomes.

\section{Conclusions}

In conclusion, some subtle differences in CMR imaging are present between PPCM patients and women with DCM. Most importantly, the right atrium is larger and more impaired in PPCM in comparison to DCM, and the left ventricular global longitudinal strain is less reduced in PPCM than in DCM. Furthermore, similarly to DCM, PPCM patients with LGE on CMR have more dilated and impaired left and right ventricles than compared to patients without LGE. The results of this study provide new insights into the cardiac phenotype of PPCM in comparison to DCM and may thereby provide valuable information to complement the clinical findings needed in establishing a diagnosis of PPCM. This study also shows that CMR can be a useful and robust tool to help differentiate between PPCM and DCM if the clinical picture is ambiguous.

Supplementary Materials: The following are available online at https:/ / www.mdpi.com/article/10 .3390 /diagnostics11101752/s1, Table S1: Intraobserver and interobserver reproducibility of feature tracking. 
Author Contributions: Conceptualization, J.P.-M., K.K. and Ł.M.; data curation, J.P.-M., K.K., Ł.M., B.M.-W., M.Ś., M.M. and J.H.; formal analysis, J.P.-M., Ł.M., B.M.-W., M.Ś., M.M. and J.H.; funding acquisition, J.P.-M. and Ł.M.; investigation, J.P.-M., K.K., Ł.M., B.M.-W., M.Ś., M.M., J.H., J.G., M.D. and Z.D.; methodology, J.P.-M., K.K., Ł.M., B.M.-W., M.Ś., M.M., J.H., J.G., M.D. and Z.D.; project administration, J.P.-M., K.K. and Ł.M.; resources, J.P.-M., K.K., Ł.M., M.Ś. and M.M.; software, J.P.-M.; supervision, J.P.-M., M.M., J.G., M.D. and Z.D.; validation, J.P.-M.; visualization, J.P.-M.; writingoriginal draft, J.P.-M.; writing—review and editing, J.P.-M., K.K., Ł.M., B.M.-W., M.Ś., M.M., J.H., J.G., M.D. and Z.D. All authors have read and agreed to the published version of the manuscript.

Funding: This research was funded by The National Institute of Cardiology (statutory grants no. 2.28/VII/17 received by Ł.M.).

Institutional Review Board Statement: The study was conducted according to the guidelines of the Declaration of Helsinki and approved by the Institutional Ethics Committee of The National Institute of Cardiology (protocol IK-NPIA-0021-115/1621/2019, date of approval 8 October 2019, and protocol IK-NPIA-0021-100/1469/18, date of approval 21 December 2018).

Informed Consent Statement: Informed consent was obtained from all subjects involved in the study.

Data Availability Statement: The data presented in this study are available upon request from the corresponding author.

Conflicts of Interest: The authors declare no conflict of interest.

\section{References}

1. Bollen, I.A.; Ehler, E.; Fleischanderl, K.; Bouwman, F.; Kempers, L.; Ricke-Hoch, M.; Hilfiker-Kleiner, D.; Dos Remedios, C.G.; Krüger, M.; Vink, A.; et al. Myofilament Remodeling and Function Is More Impaired in Peripartum Cardiomyopathy Compared with Dilated Cardiomyopathy and Ischemic Heart Disease. Am. J. Pathol. 2017, 187, 2645-2658. [CrossRef]

2. van Hoeven, K.; Kitsis, R.N.; Katz, S.D.; Factor, S.M. Peripartum versus idiopathic dilated cardiomyopathy in young women-A comparison of clinical, pathologic and prognostic features. Int. J. Cardiol. 1993, 40, 57-65. [CrossRef]

3. Pillarisetti, J.; Kondur, A.; Alani, A.; Reddy, M.; Vacek, J.; Weiner, C.P.; Ellerbeck, E.; Schreiber, T.; Lakkireddy, D. Peripartum Cardiomyopathy. J. Am. Coll. Cardiol. 2014, 63, 2831-2839. [CrossRef]

4. Kryczka, K.E.; Demkow, M.; Dzielinska, Z. Peripartum cardiomyopathy-A cardiovascular disease in pregnancy and puerperi-um. The actual state of knowledge, challenges, and perspectives. Ginekol. Pol. 2020, 92, 147-152. [CrossRef]

5. Lane, R.E.; Cowie, M.R.; Chow, A.W. Prediction and prevention of sudden cardiac death in heart failure. Heart 2005, 91, 674-680. [CrossRef]

6. Pierce, J.A. Familial Occurrence of Postpartal Heart Failure. Arch. Intern. Med. 1963, 111, 651-655. [CrossRef]

7. Ware, J.; Julie, D.; Mazaika, E.; Yasso, C.M.; DeSouza, T.; Cappola, T.P.; Tsai, E.; Hilfiker-Kleiner, D.; Kamiya, C.A.; Mazzarotto, F.; et al. Shared Genetic Predisposition in Peripartum and Dilated Cardiomyopathies. N. Engl. J. Med. 2016, 374, 233-241. [CrossRef]

8. Van Spaendonck-Zwarts, K.Y.; Posafalvi, A.; Berg, M.V.D.; Hilfiker-Kleiner, D.; Bollen, I.A.; Sliwa, K.; Alders, M.; Almomani, R.; Van Langen, I.M.; Van Der Meer, P.; et al. Titin gene mutations are common in families with both peripartum cardiomyopathy and dilated cardiomyopathy. Eur. Heart J. 2014, 35, 2165-2173. [CrossRef] [PubMed]

9. Petryka-Mazurkiewicz, J.; Kryczka, K.; Marona, M.; Kuriata, J.; Sitkowska-Rysiak, E.; Konopka, A.; Marczak, M.; Kołsut, P.; Kuśmierczyk, M.; Demkow, M.; et al. Biventricular assist device-induced recovery from acute heart failure in peripartum cardiomyopathy on cardiac magnetic resonance imaging. Kardiol. Pol. 2020, 78, 1284-1285. [CrossRef]

10. Mazurkiewicz, Ł.; Petryka, J.; Śpiewak, M.; Miłosz-Wieczorek, B.; Małek, Ł.A.; Jasińska, A.; Jarmus, E.; Marczak, M.; Misko, J.; Grzybowski, J. Clinical and prognostic relevancy of left ventricular trabeculation assessed by cardiac magnetic resonance in patients with dilated cardiomyopathy. Kardiol. Pol. 2017, 75, 794-803. [CrossRef]

11. Mouquet, F.; Lions, C.; De Groote, P.; Bouabdallaoui, N.; Willoteaux, S.; Dagorn, J.; Deruelle, P.; Lamblin, N.; Bauters, C.; Beregi, J.P. Characterisation of peripartum cardiomyopathy by cardiac magnetic resonance imaging. Eur. Radiol. 2008, 18, 2765-2769. [CrossRef]

12. Sliwa, K.; Hilfiker-Kleiner, D.; Petrie, M.C.; Mebazaa, A.; Pieske, B.; Buchmann, E.; Regitz-Zagrosek, V.; Schaufelberger, M.; Tavazzi, L.; Van Veldhuisen, D.J.; et al. Current state of knowledge on aetiology, diagnosis, management, and therapy of peripartum cardiomyopathy: A position statement from the Heart Failure Association of the European Society of Cardiology Working Group on peripartum cardiomyopathy. Eur. J. Heart Fail. 2010, 12, 767-778. [CrossRef]

13. Iles, L.M.; Ellims, A.H.; Llewellyn, H.; Hare, J.L.; Kaye, D.M.; McLean, C.A.; Taylor, A. Histological validation of cardiac magnetic resonance analysis of regional and diffuse interstitial myocardial fibrosis. Eur. Heart J. Cardiovasc. Imaging 2015, 16, 14-22. [CrossRef] 
14. Mazurkiewicz, Ł.; Petryka, J.; Spiewak, M.; Miłosz-Wieczorek, B.; Werys, K.; Małek, Ł.A.; Polanska-Skrzypczyk, M.; Ojrzynska, N.; Kubik, A.; Marczak, M.; et al. Biventricular mechanics in prediction of severe myocardial fibrosis in patients with dilated cardiomyopathy: CMR study. Eur. J. Radiol. 2017, 91, 71-81. [CrossRef]

15. Mazurkiewicz, Ł.; Ziółkowska, L.; Petryka, J.; Śpiewak, M.; Łukasz, M.; Kubik, A.; Marczak, M.; Misko, J.; Brzezińska-Rajszys, G. Biatrial performance in children with hypertrophic cardiomyopathy: CMR study. Eur. Radiol. 2018, 28, 5148-5159. [CrossRef] [PubMed]

16. Gulati, A.; Ismail, T.F.; Jabbour, A.; Alpendurada, F.; Guha, K.; Ismail, N.A.; Raza, S.; Khwaja, J.; Brown, T.D.; Morarji, K.; et al. The Prevalence and Prognostic Significance of Right Ventricular Systolic Dysfunction in Nonischemic Dilated Cardiomyopathy. Circulation 2013, 128, 1623-1633. [CrossRef] [PubMed]

17. Haghikia, A.; Röntgen, P.; Vogel-Claussen, J.; Schwab, J.; Westenfeld, R.; Ehlermann, P.; Berliner, D.; Podewski, E.; Hilfiker-Kleiner, D.; Bauersachs, J. Prognostic implication of right ventricular involvement in peripartum cardiomyopathy: A cardiovascular magnetic resonance study. ESC Heart Fail. 2015, 2, 139-149. [CrossRef]

18. Spiewak, M.; Małek, Ł.A.; Petryka, J.; Mazurkiewicz, L.; Marczak, M.; Biernacka, E.K.; Kowalski, M.; Hoffman, P.; Demkow, M.; Miśko, J.; et al. Determinants of left- and right?ventricular ejection fractions in patients with repaired tetralogy of Fallot: A cardiac magnetic resonance imaging study. Pol. Arch. Intern. Med. 2013, 123, 539-546. [CrossRef]

19. Lang, R.M.; Bierig, M.; Devereux, R.B.; Flachskampf, F.A.; Foster, E.; Pellikka, P.A.; Picard, M.; Roman, M.J.; Seward, J.; Shanewise, J.S.; et al. Recommendations for Chamber Quantification: A Report from the American Society of Echocardiography's Guidelines and Standards Committee and the Chamber Quantification Writing Group, Developed in Conjunction with the European Association of Echocardiography, a Branch of the European Society of Cardiology. J. Am. Soc. Echocardiogr. 2005, 18, 1440-1463. [CrossRef]

20. Anderson, J.; Horne, B.; Pennell, D. Atrial Dimensions in Health and Left Ventricular Disease Using Cardiovascular Magnetic Resonance. J. Cardiovasc. Magn. Reson. 2005, 7, 671-675. [CrossRef]

21. Pritchett, A.M.; Mahoney, D.W.; Jacobsen, S.; Rodeheffer, R.J.; Karon, B.L.; Redfield, M.M. Diastolic dysfunction and left atrial volume: A population-based study. J. Am. Coll. Cardiol. 2005, 45, 87-92. [CrossRef]

22. Gulati, A.; Ismail, T.F.; Jabbour, A.; Ismail, N.; Morarji, K.; Ali, A.; Raza, S.; Khwaja, J.; Brown, T.D.; Liodakis, E.; et al. Clinical utility and prognostic value of left atrial volume assessment by cardiovascular magnetic resonance in non-ischaemic dilated cardiomyopathy. Eur. J. Heart Fail. 2013, 15, 660-670. [CrossRef]

23. Doherty, N.E.; Seelos, K.C.; Suzuki, J.-I.; Caputo, G.R.; O’Sullivan, M.; Sobol, S.M.; Cavero, P.; Chatterjee, K.; Parmley, W.W.; Higgins, C.B. Application of cine nuclear magnetic resonance imaging for sequential evaluation of response to angiotensinconverting enzyme inhibitor therapy in dilated cardiomyopathy. J. Am. Coll. Cardiol. 1992, 19, 1294-1302. [CrossRef]

24. Bellenger, N.; Davies, L.C.; Francis, J.; Coats, A.S.; Pennell, D. Reduction in Sample Size for Studies of Remodeling in Heart Failure by the Use of Cardiovascular Magnetic Resonance. J. Cardiovasc. Magn. Reson. 2000, 2, 271-278. [CrossRef]

25. Marmursztejn, J.; Vignaux, O.; Goffinet, F.; Cabanes, L.; Duboc, D. Delayed-enhanced cardiac magnetic resonance imaging features in peripartum cardiomyopathy. Int. J. Cardiol. 2009, 137, e63-e64. [CrossRef]

26. Arora, N.P.; Mahajan, N.; Mohamad, T.; Kottam, A.; Afonso, L.C.; Danrad, R.; Li, T. Cardiac Magnetic Resonance Imaging in Peripartum Cardiomyopathy. Am. J. Med. Sci. 2014, 347, 112-117. [CrossRef] [PubMed]

27. Leurent, G.; Baruteau, A.; Larralde, A.; Ollivier, R.; Schleich, J.; Boulmier, D.; Bedossa, M.; Langella, B.; Le Breton, H. Contribution of cardiac MRI in the comprehension of peripartum cardiomyopathy pathogenesis. Int. J. Cardiol. 2009, 132, e91-e93. [CrossRef] [PubMed]

28. Wu, K.C.; Weiss, R.G.; Thiemann, D.R.; Kitagawa, K.; Schmidt, A.; Dalal, D.; Lai, S.; Bluemke, D.; Gerstenblith, G.; Marbán, E.; et al. Late Gadolinium Enhancement by Cardiovascular Magnetic Resonance Heralds an Adverse Prognosis in Nonischemic Cardiomyopathy. J. Am. Coll. Cardiol. 2008, 51, 2414-2421. [CrossRef]

29. Mewton, N.; Liu, C.Y.; Croisille, P.; Bluemke, D.; Lima, J.A. Assessment of Myocardial Fibrosis with Cardiovascular Magnetic Resonance. J. Am. Coll. Cardiol. 2011, 57, 891-903. [CrossRef]

30. Ntusi, N.B.A.; Chin, A. Characterisation of peripartum cardiomyopathy by cardiac magnetic resonance imaging. Eur. Radiol. 2009, 19, 1324-1325. [CrossRef]

31. Buss, S.J.; Breuninger, K.; Lehrke, S.; Voss, A.; Galuschky, C.; Lossnitzer, D.; Andre, F.; Ehlermann, P.; Franke, J.; Taeger, T.; et al. Assessment of myocardial deformation with cardiac magnetic resonance strain imaging improves risk stratification in patients with dilated cardiomyopathy. Eur. Heart J. Cardiovasc. Imaging 2015, 16, 307-315. [CrossRef] [PubMed]

32. Sugahara, M.; Kagiyama, N.; Hasselberg, N.E.; Blauwet, L.A.; Briller, J.; Cooper, L.; Fett, J.D.; Hsich, E.; Wells, G.; McNamara, D.; et al. Global Left Ventricular Strain at Presentation Is Associated with Subsequent Recovery in Patients with Peripartum Cardiomyopathy. J. Am. Soc. Echocardiogr. 2019, 32, 1565-1573. [CrossRef] [PubMed]

33. Briasoulis, A.; Mocanu, M.; Marinescu, K.; Qaqi, O.; Palla, M.; Telila, T.; Afonso, L. Longitudinal systolic strain profiles and outcomes in peripartum cardiomyopathy. Echocardiography 2016, 33, 1354-1360. [CrossRef] [PubMed]

34. Cameli, M.; Mondillo, S.; Righini, F.M.; Lisi, M.; Dokollari, A.; Lindqvist, P.; Maccherini, M.; Henein, M. Left Ventricular Deformation and Myocardial Fibrosis in Patients With Advanced Heart Failure Requiring Transplantation. J. Card. Fail. 2016, 22, 901-907. [CrossRef] [PubMed] 